\title{
A NORMAL FORM IN THE HOMEOTOPY GROUP OF A SURFACE OF GENUS 2, WITH APPLICATIONS TO 3-MANIFOLDS
}

\author{
JOAN S. BIRMAN
}

\begin{abstract}
It is shown that elements in the homeotopy group of a closed, compact, orientable 2-manifold of genus 2 can be put into a unique normal form which allows them to be enumerated systematically. As an application, the class of 3-manifolds which admit Heegaard splittings of genus 2 are shown to be denumerable, and a procedure is given for enumerating presentations for their fundamental groups.
\end{abstract}

1. Introduction. Let $T$ be a closed, compact orientable 2-manifold of genus 2, and let $H$ be its homeotopy group, that is, the group of all homeomorphisms of $T \rightarrow T$ modulo the subgroup of those homeomorphisms which are isotopic to the identity. Generators and defining relations for the group $H$ (or rather a subgroup of index 2 in $H$ ) were determined in [1]. We will show here that the elements in $H$ can be put into a unique normal form which shows that they are denumerable, and we will give an algorithm for enumerating them systematically.

This result has potential applications in the study of 3-manifolds. Define a "genus 2 Heegaard splitting" to be a compact 3-manifold $M$ without boundary which admits a decomposition as $M=X \cup X^{\prime}$, where $X$ and $X^{\prime}$ are solid handle-bodies of genus 2, and $X \cap X^{\prime}=\mathrm{bd} X=\mathrm{bd} X^{\prime}$. Each such splitting can be associated (nonuniquely) with an element in $H$, so that our enumeration of the elements in $H$ is equivalent to an enumeration (with duplications) of all possible genus 2 Heegaard splittings. We show how to use our enumeration to enumerate presentations (in terms of 2-generators, 2 relators) of the fundamental groups of all genus 2 Heegaard splittings. This enumeration might be useful as a tool in studying the classification of 3-manifolds.

At the conclusion we discuss briefly why the approach used here does not generalize to genus $>2$.

Received by the editors August 16, 1971.

AMS 1970 subject classifications. Primary 20E40, 20F05, 20F10, 20F55, 55A05, 55A99; Secondary 57E30.

Key words and phrases. Mapping class group, homeotopy group, braid group, Heegaard splitting, closed compact orientable surface.

(C) American Mathematical Society 1972 
2. Enumerating the elements in $H$. Our enumeration will be based on the following reasoning: we will first show that the structure of $H$ is closely related to the structure of the 5 -string Artin braid group, $B_{5}$. We will then use results due to F. Garside [3] on the braid group to develop a normal form in $H$.

Instead of $H$, we first consider the mapping class group $M$ of the surface $T$, where $M$ is defined to be the subgroup of $H$ in which admissible maps are restricted to those homeomorphisms of $T \rightarrow T$ which preserve orientation. Using the well-known fact that the group $H$ is isomorphic to Aut $\pi_{1} T / \operatorname{Inn} \pi_{1} T$, we can interpret elements of $H$ and $M$ as classes of automorphisms of the fundamental group $\pi_{1} T$ of the surface $T$. Let $\pi_{1} T$ be presented in terms of canonical generators $a_{1}, b_{1}, a_{2}, b_{2}$ which satisfy the single relation $\left[a_{1}, b_{1}\right]\left[a_{2}, b_{2}\right]=1$. Let $\tau_{1}, \cdots, \tau_{5}$ be the automorphism classes of:

$$
\begin{aligned}
\tau_{1}: a_{1} & \rightarrow a_{1} b_{1}^{-1}, \\
\tau_{2}: b_{1} & \rightarrow b_{1} a_{1}, \\
\tau_{3}: a_{1} & \rightarrow a_{1} b_{1}^{-1} a_{2} b_{2} a_{2}^{-1}, \\
a_{2} & \rightarrow a_{2} b_{2}^{-1} a_{2}^{-1} b_{1} a_{2}, \\
b_{1} & \rightarrow a_{2} b_{2}^{-1} a_{2}^{-1} b_{1} a_{2} b_{2} a_{2}^{-1}, \\
\tau_{4}: b_{2} & \rightarrow b_{2} a_{2}, \\
\tau_{5}: a_{2} & \rightarrow a_{2} b_{2}^{-1},
\end{aligned}
$$

where all generators which are not listed explicitly are assumed to be kept fixed. Then $M$ admits the presentation [1]:

generators: $\tau_{1}, \cdots, \tau_{5}$,

relations:

$$
\begin{aligned}
{\left[\tau_{i}, \tau_{j}\right] } & =1 \quad \text { if }|i-j| \geqq 2,1 \leqq i, j \leqq 5, \\
\tau_{i} \tau_{i+1} \tau_{i} & =\tau_{i+1} \tau_{i} \tau_{i+1}, \quad 1 \leqq i \leqq 4, \\
\left(\tau_{1} \tau_{2} \tau_{3} \tau_{4} \tau_{5}\right)^{6} & =1, \\
\left(\tau_{1} \tau_{2} \tau_{3} \tau_{4} \tau_{5}^{2} \tau_{4} \tau_{3} \tau_{2} \tau_{1}\right)^{2} & =1, \\
{\left[\tau_{1} \tau_{2} \tau_{3} \tau_{4} \tau_{5}^{2} \tau_{4} \tau_{3} \tau_{2} \tau_{1}, \tau_{i}\right] } & =1, \quad 1 \leqq i \leqq 5
\end{aligned}
$$

The group $H$ is an extension of $M$ by a cyclic group or order 2. Define a new element $\tau_{6} \in H$ by the rule:

$$
\begin{aligned}
\tau_{6}: a_{1} & \rightarrow b_{1}, \\
a_{2} & \rightarrow b_{2}, \\
b_{1} & \rightarrow a_{1}, \\
b_{2} & \rightarrow a_{2} .
\end{aligned}
$$


Since $\tau_{6}$ is orientation-reversing, the pair $\left(1, \tau_{6}\right)$ can be used as coset representatives for $M$ in $H$. Thus if we can show that the elements in $M$ are denumerable, it will follow that those in $H$ are also denumerable, with each element $x \in M$ corresponding to two elements $x$ and $x \tau_{6}$ in $H$.

To develop a normal form for elements in $M$, we first observe that it follows from relations (2)-(6) that the element $\alpha=\tau_{1} \tau_{2} \tau_{3} \tau_{4} \tau_{5}^{2} \tau_{4} \tau_{3} \tau_{2} \tau_{1}$ is in the center of $M$ and has order 2 . Let $G$ be the quotient group obtained by setting $\alpha=1$, and let $\rho_{i} \in G$ be the image of $\tau_{i} \in M$ under the homomorphism from $M \rightarrow G$. Then $G$ is generated by $\rho_{1}, \cdots, \rho_{5}$ and has defining relations $(2)_{\rho},(3)_{\rho},(4)_{\rho}$ and:

$$
\rho_{1} \rho_{2} \rho_{3} \rho_{4} \rho_{5}^{2} \rho_{4} \rho_{3} \rho_{2} \rho_{1}=1
$$

where $(2)_{\rho},(3)_{\rho},(4)_{\rho}$ mean relations (2), (3), (4) but with the $\rho$ 's substituted for the $\tau$ 's. If we could list elements in $G$, we could list elements in $M$, by lifting the element $\rho_{\mu_{1}}^{\varepsilon_{1}} \cdots \rho_{\mu_{r}}^{\varepsilon_{r}}$ in $G$ to the pair $\tau_{\mu_{1}}^{\varepsilon_{1}} \cdots \tau_{\mu_{r}}^{\varepsilon_{r}}$ and $\alpha \tau_{\mu_{1}}^{\varepsilon_{1}} \cdots \tau_{\mu_{r}}^{\varepsilon_{1}}$ in $M$.

Now we note that if we add to relations $(2)_{\rho},(3)_{\rho},(4)_{\rho}$ and (8) the additional relation that the square of every generator is trivial, then our presentation for $G$ will go over to a presentation for the symmetric group $\Sigma_{6}$ on 6 letters [2, equation 6.28], each $\rho_{i}$ being mapped onto the transposition $(i, i+1)$. Let $K$ be the subgroup of $G$ consisting of all elements in $G$ whose images in $\Sigma_{6}$ leave the letter 6 invariant. Then $K$ has index 6 in $G$, and each element $\beta \in G$ has a unique representation $\beta=\gamma k_{\mu}$, where $\gamma \in K$ and the elements $k_{0}, \cdots, k_{6}$ are coset representatives for $K$ in $G$, with

$$
k_{0}=1, \quad k_{\mu}=\rho_{5} \rho_{4} \cdots \rho_{\mu} \text { if } \mu=1, \cdots, 5 .
$$

Thus our enumeration is reduced to an enumeration in $K$. Since the elements $k_{0}, k_{1}, \cdots, k_{5}$ are a Schreier set of coset representatives for $K$ in $G$, we can apply the Schreier-Reidemeister method [4] to the known presentation for $G$ to obtain a presentation for $K$. This calculation gives that $K$ is generated by $\rho_{1}, \rho_{2}, \rho_{3}, \rho_{4}$ and has defining relations:

$$
\begin{gathered}
\rho_{i} \rho_{j}=\rho_{j} \rho_{i}, \quad 1 \leqq i, j \leqq 4,|i-j| \geqq 2, \\
\rho_{i} \rho_{i+1} \rho_{i}=\rho_{i+1} \rho_{i} \rho_{i+1}, \quad 1 \leqq i \leqq 4, \\
\left(\rho_{1} \rho_{2} \rho_{3} \rho_{4}\right)^{5}=1 .
\end{gathered}
$$

To understand the group $K$, we compare it to a group which is closely related to it, the 5-string Artin braid group, $B_{5}$. The $n$-string braid group $B_{n}$ can be defined as the abstract group with generators $\sigma_{1}, \cdots, \sigma_{n-1}$ and defining relations $(9)_{\sigma}$ and $(10)_{\sigma}$ where the indices $i, j$ are allowed to run between 1 and $n-1$ instead of between 1 and 4 . Now, the element 
$\left(\sigma_{1} \sigma_{2} \cdots \sigma_{n-1}\right)^{n}$ generates the center $Z\left(B_{n}\right)$ of $B_{n}$ (see, for example, [3]). Hence every element $\delta \in B_{n}$ can be written uniquely in the form

$$
\delta=\left(\sigma_{1} \sigma_{2} \cdots \sigma_{n-1}\right)^{n m} V\left(\sigma_{1}, \cdots, \sigma_{n-1}\right)
$$

where $m$ is an integer. Under the natural homomorphism from $B_{5} \rightarrow K$ the element $\left(\sigma_{1} \sigma_{2} \sigma_{3} \sigma_{4}\right)^{5 m} V\left(\sigma_{1}, \sigma_{2}, \sigma_{3}, \sigma_{4}\right)$ in $B_{5}$ goes over to the element $V\left(\rho_{1}, \rho_{2}, \rho_{3}, \rho_{4}\right)$ in $K$, where two words $V$ and $V^{\prime}$ in $K$ will be equivalent iff their preimages in $B_{5}$ are equivalent for some fixed $m$. Thus if we can develop a scheme for enumerating the distinct coset representatives of the center $Z\left(B_{5}\right)$ in $B_{5}$, we will also be able to enumerate the distinct elements of $K$, which will in turn enable us to enumerate elements in $G$, in $M$, and finally in $H$.

The braid group $B_{n}$ was studied by Garside in [3]. Garside developed a normal form for words in $B_{n}$, which we claim allows us to enumerate the coset representatives of $Z\left(B_{n}\right)$. In $B_{n}$, define the elements:

$$
\Delta=\left(\sigma_{1} \cdots \sigma_{n-1}\right)\left(\sigma_{1} \cdots \sigma_{n-2}\right) \cdots\left(\sigma_{1} \sigma_{2}\right)\left(\sigma_{1}\right), \quad \phi=\left(\sigma_{1} \cdots \sigma_{n-1}\right)^{n} .
$$

The element $\phi$ generates $Z\left(B_{n}\right)$. Moreover, as a consequence of relations $(9)_{\sigma}$ and $(10)_{\sigma}$ one can show that in $B_{n}$ :

$$
\Delta^{2}=\phi
$$

Now, Garside finds coset representatives for the infinite cyclic subgroup of $B_{n}$ generated by $\Delta$. If we denote his coset representatives by $\left\{P_{i}, i \in I\right\}$, then it is immediate that $\left\{P_{i}, \Delta P_{i} ; i \in I\right\}$ will be a set of coset representatives for $Z\left(B_{n}\right)$. So we need only show that Garside's coset representatives are denumerable.

The words $P_{i}$ obtained by Garside have a very special form which we describe briefly. Let $S_{n}$ be the semigroup with generators $\sigma_{1}, \cdots, \sigma_{n-1}$ and with the same defining relations as $B_{n}$. Garside shows that $S_{n}$ can be imbedded in $B_{n}$, and that the coset representatives $\left\{P_{i}, i \in I\right\}$ can be chosen to be in $S_{n}$, i.e. so that they involve only positive powers of the $\sigma_{i}$. He also gives a procedure for testing whether a given $P\left(\sigma_{1}, \cdots, \sigma_{n-1}\right) \in S_{n}$ is a coset representative (i.e. is "prime to $\Delta$ " and is "lexicographically smallest" in the "diagram of $P$ " in Garside's notation; for details see [3]). Garside's procedure for finding the coset representative of a given word goes over immediately to a procedure for listing coset representatives: one simply lists all words in $S_{n}$ in order of increasing length, finds the coset representative of each such word, and enters it in the list if it is not a coset representative which has appeared earlier on the list.

It then follows that the elements of our original group $H$ are also denumerable, because each coset representative $P\left(\sigma_{1}, \sigma_{2}, \sigma_{3}, \sigma_{4}\right)$ of the 
cyclic subgroup $\langle\Delta\rangle$ in $B_{5}$ corresponds to precisely 48 distinct words $W\left(\tau_{1}, \cdots, \tau_{6}\right) \in H$, i.e., the words:

where:

$$
W\left(\tau_{1}, \cdots, \tau_{6}\right)=\alpha^{\varepsilon_{1}} \Delta^{\varepsilon_{2}} P\left(\tau_{1}, \tau_{2}, \tau_{3}, \tau_{4}\right) k_{\mu} \tau_{6}^{\varepsilon_{3}}
$$

$$
\begin{array}{cl}
\alpha=\tau_{1} \tau_{2} \tau_{3} \tau_{4} \tau_{5}^{2} \tau_{4} \tau_{3} \tau_{2} \tau_{1}, & \Delta=\tau_{1} \tau_{2} \tau_{3} \tau_{4} \tau_{1} \tau_{2} \tau_{3} \tau_{1} \tau_{2} \tau_{1}, \\
\mu=0,1,2,3,4,5, & \\
k_{\mu}=\tau_{5} \tau_{4} \cdots \tau_{\mu} & \text { if } \mu=1,2,3,4,5, \\
=1 \quad & \text { if } \mu=0, \\
\varepsilon_{1}, \varepsilon_{2}, \varepsilon_{3}=0 & \text { or } 1 .
\end{array}
$$

3. Enumerating the fundamental groups of the genus 2 Heegaard splittings. Let $\tau \in H$, and suppose that $\tau$ is represented by an automorphism which acts on $\pi_{1} T$ as follows:

$$
\begin{aligned}
\tau: a_{i} & \rightarrow A_{i}\left(a_{1}, a_{2}, b_{1}, b_{2}\right), \\
b_{i} & \rightarrow B_{i}\left(a_{1}, a_{2}, b_{1}, b_{2}\right), \quad i=1,2 .
\end{aligned}
$$

Then it is shown in [5, Theorem 10.1] that a genus 2 Heegaard splitting exists with fundamental group:

$$
\pi_{1}\left(X \cup X^{\prime}\right)=\left\langle a_{1}, a_{2} ; A_{i}\left(a_{1}, a_{2}, 1,1\right), i=1,2\right\rangle .
$$

The enumeration procedure described in $\$ 2$ can be used to obtain an enumeration of the relators $A_{i}\left(a_{1}, a_{2}, 1,1\right), i=1,2$, by using equation (1) and (7) to compute the action of $\tau$ on the elements $a_{1}, a_{2} \in \pi_{1} T$ for each $\tau \in H$. Thus we could enumerate the fundamental groups of all 3-manifolds which admit genus 2 Heegaard splittings. Of course there will be duplications, both because the same words $A_{1}$ and $A_{2}$ will appear repeatedly, and more seriously because no procedure exists for distinguishing isomorphism classes of groups which are defined by presentations.

4. Generalizing to genus $>2$. The analysis given above does not carry over to genus $>2$, because it depends crucially on the presentation obtained in [1] for $M$, which generalizes to a presentation for a proper subgroup of the mapping class group $M\left(T_{g}\right)$ of a closed, compact orientable surface $T_{g}$ of genus $g>2$. The algebraic relationship between this subgroup and the full group $M\left(T_{g}\right)$ is not, to the author's knowledge, well understood.

5. Acknowledgement. The author wishes to thank Ralph Tindell for stimulating discussions relating to the problem of classification of 3manifolds. 


\section{REFERENCES}

1. J. Birman and H. Hilden, Mapping class groups of closed surfaces as covering spaces, Ann. of Math. Studies, no. 66, Princeton Univ. Press, Princeton, N.J., 1971.

2. H. S. M. Coxeter and W. O. J. Moser, Generators and relations for discrete groups, 2nd ed., Ergebnisse der Mathematik und ihrer Grenzgebiete, N.F., Band 14, SpringerVerlag, Berlin and New York, 1965. MR 30 \#4818.

3. F. A. Garside, The braid group and other groups, Quart. J. Math. Oxford Ser. (2) 20 (1969), 235-254. MR 40 \#2051.

4. W. Magnus, A. Karrass and D. Solitar, Combinatorial group theory: Presentations of groups in terms of generators and relations, Pure and Appl. Math., vol. 13, Interscience, New York, 1966. MR 34 \#7617.

5. C. D. Papakyriakopoulos, A reduction of the Poincaré conjecture to group theoretic conjectures, Ann. of Math (2) 77 (1963), 250-305. MR 26 \#3027.

Department of Mathematics, Stevens Institute of Technology, Hoboken, NEW JERSEY 07030 